\title{
On Neumann and Poincare Problems in $A$-harmonic Analysis
}

\author{
Artyem Yefimushkin \\ Institute of Applied Mathematics and Mechanics, National Academy of Sciences of Ukraine \\ Email: a.yefimushkin@gmail.com
}

\begin{abstract}
The existence of nonclassical solutions is proved for the Neumann and Poincare problems for generalizations of the Laplace equation in anisotropic and nonhomogeneous media in almost smooth domains with arbitrary boundary data that are measurable with respect to logarithmic capacity. Moreover, it is shown that the spaces of such solutions have the infinite dimension.
\end{abstract}

Keywords: Neumann problem, Poincare problem, $A$-harmonic functions, logarithmic capacity, anisotropic and nonhomogeneous media.

\section{Introduction}

The classical boundary value problems of the theory of analytic functions, such as the Dirichlet, Hilbert, Riemann, Neumann and Poincare problems, take a fundamental part in contemporary analysis and applications to actual problems of mathematical physics. Note that these boundary value problems are closely interconnected, see e.g. $[1,2,3,4,5,6]$. In this paper we continue the development of the theory of the above boundary value problems for analytic functions in Jordan domains with measurable boundary data and extend the theory to the more general class of quasiconformal functions.

It is well-known that the Neumann problem for Laplace equation has no classical solutions, generally speaking even for some continuous boundary data, see e.g. [7]. The main goal of this paper is to show that the Neumann problem with arbitrary boundary data that are measurable with respect to logarithmic capacity has nonclassical solutions for the Laplace equation as well as for its generalizations. The result is based on a reduction of this problem to the Riemann-Hilbert boundary value problem whose solution was recently obtained in [6].

First of all recall a more general problem on directional derivatives for the harmonic functions in the unit disk $\mathbb{D}=\{z \in \mathbb{C}:|z|<1\}, z=x+i y$. The classic setting of the latter problem is to find a function $u: \mathbb{D} \rightarrow \mathbb{R}$ that is twice continuously differentiable, admits a continuous extension to the boundary of $\mathbb{D}$ together with its first partial derivatives, satisfies the Laplace equation

$$
\Delta u:=\frac{\partial^{2} u}{\partial x^{2}}+\frac{\partial^{2} u}{\partial y^{2}}=0 \quad \forall z \in \mathbb{D}
$$

and the boundary condition with a prescribed continuous date $\varphi: \partial \mathbb{D} \rightarrow \mathbb{R}$ :

$$
\frac{\partial u}{\partial \nu}=\varphi(\zeta) \quad \forall \zeta \in \partial \mathbb{D}
$$

where $\frac{\partial u}{\partial \nu}$ denotes the derivative of $u$ at $\zeta$ in a direction $\nu=\nu(\zeta),|\nu(\zeta)|=1$ :

$$
\frac{\partial u}{\partial \nu}:=\lim _{t \rightarrow 0} \frac{u(\zeta+t \cdot \nu)-u(\zeta)}{t} .
$$

The Neumann problem is a special case of the above problem on directional derivatives with the boundary condition

$$
\frac{\partial u}{\partial n}=\varphi(\zeta) \quad \forall \zeta \in \partial \mathbb{D}
$$

where $n$ denotes the unit interior normal to $\partial \mathbb{D}$ at the point $\zeta$. 
In turn, the above problem on directional derivatives is a special case of the Poincare problem with the boundary condition

$$
a \cdot u+b \cdot \frac{\partial u}{\partial \nu}=\varphi(\zeta) \quad \forall \zeta \in \partial \mathbb{D}
$$

where $a=a(\zeta)$ and $b=b(\zeta)$ are real-valued functions given on $\partial \mathbb{D}$.

Recall also that twice continuously differentiable solutions of the Laplace equation are called harmonic functions. As known, such functions are infinitely differentiable and they are real and imaginary parts of analytic functions.

\section{Definitions and Preliminary Remarks}

The partial differential equations in the divergence form below take a significant part in many problems of mathematical physics, in particular, in anisotropic and inhomogeneous media. These equations are closely interconnected with Beltrami equations, see e.g. [1].

Let $D$ be a domain in the complex plane $\mathbb{C}$ and let $\mu: D \rightarrow \mathbb{C}$ be a measurable function with $|\mu(z)|<1$ a.e. Recall that a partial differential equation

$$
f_{\bar{z}}=\mu(z) \cdot f_{z}
$$

where $f_{\bar{z}}=\bar{\partial} f=\left(f_{x}+i f_{y}\right) / 2, f_{z}=\partial f=\left(f_{x}-i f_{y}\right) / 2, z=x+i y, f_{x}$ and $f_{y}$ are partial derivatives of the function $f$ in $x$ and $y$, respectively, is said to be a Beltrami equation. The Beltrami equation (1) is said to be nondegenerate if $\|\mu\|_{\infty}<1$.

In this connection, note that if $f=u+i \cdot v$ is a regular solution of the Beltrami equation (1), then the function $u$ is a continuous generalized solution of the divergence-type equation

$$
\operatorname{div} A(z) \nabla u=0,
$$

called A-harmonic function, i.e. $u \in C \cap W^{1,1}$ and

$$
\int_{D}\langle A(z) \nabla u, \nabla \varphi\rangle=0 \quad \forall \varphi \in C_{0}^{\infty}(D),
$$

where $A(z)$ is the matrix function:

$$
A=\left(\begin{array}{cc}
\frac{|1-\mu|^{2}}{1-|\mu|^{2}} & \frac{-2 \operatorname{Im} \mu}{1-|\mu|^{2}} \\
\frac{-2 \operatorname{Im} \mu}{1-|\mu|^{2}} & \frac{|1+\mu|^{2}}{1-|\mu|^{2}}
\end{array}\right)
$$

As we see in (3), the matrix $A(z)$ is symmetric and its entries $a_{i j}=a_{i j}(z)$ are dominated by the quantity

$$
K_{\mu}(z)=\frac{1+|\mu(z)|}{1-|\mu(z)|}
$$

and, thus, they are bounded if the Beltrami equation (1) is not degenerate.

Vice verse, uniformly elliptic equations (2) with symmetric $A(z)$ and $\operatorname{det} A(z) \equiv 1$ just correspond to nondegenerate Beltrami equations (1) with coefficient

$$
\mu=\frac{1}{\operatorname{det}(I+A)}\left(a_{22}-a_{11}-2 i a_{21}\right)=\frac{a_{22}-a_{11}-2 i a_{21}}{1+\operatorname{Tr} A+\operatorname{det} A},
$$

where $I$ denotes identity $2 \times 2$ matrix $\operatorname{Tr} A=a_{22}+a_{11}$, see e.g. theorem 16.1.6 in [1]. Following [2], call all such matrix functions $A(z)$ of the class $\mathcal{B}$. Recall that equation (2) is said to be uniformly elliptic, if $a_{i j} \in L^{\infty}$ and $\langle A(z) \eta, \eta\rangle \geq \varepsilon|\eta|^{2}$ for some $\varepsilon>0$ and for all $\eta \in \mathbb{R}^{2}$.

Finally, recall that homeomorphic solutions of Beltrami equations (1) of class $W_{\mathrm{loc}}^{1,1}$ is said to be quasiconformal mappings, see e.g. [8,9]. The images of the unit disk $\mathbb{D}=\{z \in \mathbb{C}:|z|<1\}$ under quasiconformal mappings of $\mathbb{C}$ onto itself are called quasidisks, and their boundaries are called quasicircles or quasiconformal curves. Recall that the bijective continuous image of a circle in $\mathbb{C}$ is called a Jordan curve. As known, any smooth (or Lipschitzian) Jordan curve is a quasiconformal curve, see e.g. point II.8.10 in $[9]$. 


\section{On Neumann and Poincare Problems for Harmonic Functions}

Let us start with the unit disk because proofs in this case are more direct and clear.

Theorem 1. Let $\nu: \partial \mathbb{D} \rightarrow \mathbb{C},|\nu(\zeta)| \equiv 1$ be a function of bounded variation, and let $\varphi: \partial \mathbb{D} \rightarrow \mathbb{R}$ be a measurable function with respect to logarithmic capacity. Then there exist harmonic functions $u: \mathbb{D} \rightarrow \mathbb{R}$ such that

$$
\lim _{z \rightarrow \zeta} \frac{\partial u}{\partial \nu}=\varphi(\zeta)
$$

along any nontangential paths for a.e. $\zeta \in \partial \mathbb{D}$ with respect to logarithmic capacity.

Proof. Indeed, by Proposition 6.1 in [6], there exists an analytic function $f: D \rightarrow \mathbb{C}$ such that

$$
\lim _{z \rightarrow \zeta} \operatorname{Re} \overline{\nu(\zeta) \cdot f(z)}=\lim _{z \rightarrow \zeta} \operatorname{Re} \nu(\zeta) \cdot f(z)=\varphi(z)
$$

along any nontangential paths for a.e. $\zeta \in \partial \mathbb{D}$ with respect to logarithmic capacity. Note that an indefinite integral $F$ of $f$ in $\mathbb{D}$ is also an analytic function and, correspondingly, the harmonic functions $u=\operatorname{Re} F$ and $v=\operatorname{Im} F$ satisfy the system of Cauchy-Riemann $v_{x}=-u_{y}$ è $v_{y}=u_{x}$. Hence

$$
f=F^{\prime}=F_{x}=u_{x}+i \cdot v_{x}=u_{x}-i \cdot u_{y}=\overline{\nabla u}
$$

where $\nabla u=u_{x}+i \cdot u_{y}$ is the gradient of the function $u$ in complex form. Thus, (5) follows from (6), i.e. $u$ is one of the desired harmonic functions because its directional derivative

$$
\frac{\partial u}{\partial \nu}=\operatorname{Re} \bar{\nu} \cdot \nabla u=\operatorname{Re} \nu \cdot \overline{\nabla u}=\langle\nu, \nabla u\rangle
$$

is the scalar product of $\nu$ and the gradient $\nabla u$ is interpreted as vectors in $\mathbb{R}^{2}$.

Remark 1. We are able to say more in the case $\operatorname{Re} n \cdot \bar{\nu}>0$ where $n=n(\zeta)$ is the unit interior normal with a tangent to $\partial \mathbb{D}$ at the point $\zeta \in \partial \mathbb{D}$. In view of $(5)$, since the limit $\varphi(\zeta)$ is finite, there is a finite limit $u(\zeta)$ of $u(z)$ as $z \rightarrow \zeta$ in $\mathbb{D}$ along the straight line passing through the point $\zeta$ and being parallel to the vector $\nu(\zeta)$ because along this line, for $z$ and $z_{0}$ that are close enough to $\zeta$,

$$
u(z)=u\left(z_{0}\right)-\int_{0}^{1} \frac{\partial u}{\partial \nu}\left(z_{0}+\tau\left(z-z_{0}\right)\right) \mathrm{d} \tau .
$$

Thus, at each point with the condition (5), there is the directional derivative

$$
\frac{\partial u}{\partial \nu}(\zeta):=\lim _{t \rightarrow 0} \frac{u(\zeta+t \cdot \nu)-u(\zeta)}{t}=\varphi(\zeta)
$$

In particular, in the case of the Neumann problem, we have by Theorem 1 and Remark 1 the following significant result.

Theorem 2. For each function $\varphi: \partial \mathbb{D} \rightarrow \mathbb{R}$ that is measurable with respect to logarithmic capacity, one can find harmonic functions $u: \mathbb{D} \rightarrow \mathbb{C}$ such that, for a.e. point $\zeta \in \partial \mathbb{D}$ with respect to logarithmic capacity, there exist:

1) the finite radial limit

$$
u(\zeta):=\lim _{r \rightarrow 1} u(r \zeta)
$$

2) the normal derivative

$$
\frac{\partial u}{\partial n}(\zeta):=\lim _{t \rightarrow 0} \frac{u(\zeta+t \cdot n)-u(\zeta)}{t}=\varphi(\zeta)
$$

3) the nontangential limit

$$
\lim _{z \rightarrow \zeta} \frac{\partial u}{\partial n}(z)=\frac{\partial f}{\partial n}(\zeta)
$$

where $n=n(\zeta)$ denotes the unit interior normal to $\partial \mathbb{D}$ at the point $\zeta$. 
Recall that a Jordan domain is called Lipschitzian if its boundary is bilipschitzian image of a circle. It is clear that such curve is rectifiable, and rectifiable curves have tangent to almost all points with respect to the length measure. A Jordan domain is called almost smooth if it is Lipschitzian and has tangent to almost all points with respect to logarithmic capacity.

Theorem 3. Let $D$ be an almost smooth Jordan domain in the complex plane $\mathbb{C}, \nu: \partial D \rightarrow \mathbb{C},|\nu(\zeta)| \equiv 1$ be a function of bounded variation and let $\varphi: \partial D \rightarrow \mathbb{R}$ be a function that is measurable with respect to logarithmic capacity. Then there exist harmonic functions $u: D \rightarrow \mathbb{R}$ such that

$$
\lim _{z \rightarrow \zeta} \frac{\partial u}{\partial \nu}=\varphi(\zeta)
$$

along any nontangential paths for a.e. point $\zeta \in \partial D$ with respect to logarithmic capacity.

Proof. The case of almost smooth Jordan domains $D$ reduces to the case of the unit disk $\mathbb{D}$ in the following way. First of all, by the Riemann theorem one can find conformal mapping $\omega$ of the domain $D$ onto $\mathbb{D}$, see e.g. Theorem II.2.1 in [10]. Then by Caratheodory theorem $\omega$ is extended to a homeomorphism of $\bar{D}$ onto $\overline{\mathbb{D}}$, see e.g. Theorem II.C.1 in [11].

As it was noted in Section 2, the boundaries of Lipschitzian domains are quasiconformal curves. Thus, by the reflection principle for quasiconformal mappings, involving the conformal reflection (inversion) with respect to the unit circle in the image and a quasiconformal reflection with respect to $\partial D$ in the preimage, we are able to extend $\omega$ to a quasiconformal mapping $\Omega: \mathbb{C} \rightarrow \mathbb{C}$, see e.g. I.8.4, II.8.2 and II.8.3 in [9]. It is clear also that $\mathcal{N}:=\left.\nu \circ \Omega^{-1}\right|_{\partial \mathbb{D}}$ is a function of bounded variation, $V_{\mathcal{N}}(\partial \mathbb{D})=V_{\nu}(\partial D)$.

The logarithmic capacity of a set coincides with its transfinite diameter, see e.g. [12] and the point 110 in [13]. Moreover, quasiconformal mappings are Hölder continuous on compacta, see e.g. Theorem II.4.3 in [9]. Hence the mappings $\Omega$ and $\Omega^{-1}$ transform sets of logarithmic capacity zero on $\partial D$ into sets of logarithmic capacity zero on $\partial \mathbb{D}$ and vice versa.

The function $\Phi:=\left.\varphi \circ \Omega^{-1}\right|_{\partial \mathbb{D}}$ is measurable with respect to logarithmic capacity. Indeed, under the given mappings any sets that are measurable with respect to logarithmic capacity are transformed into sets that are measurable with respect to logarithmic capacity because any such a set can be represented in the form of the union of a sigma-compact set and a set of logarithmic capacity zero and, under continuous mappings, the compact sets are transformed into compact sets and the latter sets are measurable with respect to logarithmic capacity.

Next, by the Lindelöf lemma $\arg [\omega(\zeta)-\omega(z)]-\arg [\zeta-z] \rightarrow$ const as $z \rightarrow \zeta$ for every point $\zeta \in \partial D$ with a tangent to $\partial D$, see e.g. Theorem II.C.2 in [11]. Hence nontangential paths in $D$ to $\zeta \in \partial D$ are transformed under the mapping $\omega$ into nontangential paths in $\mathbb{D}$ to $\xi=\omega(\zeta) \in \partial \mathbb{D}$ for a.e. $\zeta \in \partial D$ with respect to logarithmic capacity. And vice versa, nontangential paths in $\mathbb{D}$ to $\xi \in \partial \mathbb{D}$ are transformed under the mapping $\omega^{-1}$ into nontangential paths in $D$ to $\zeta=\omega^{-1}(\xi) \in \partial D$ for a.e. $\xi \in \partial \mathbb{D}$ with respect to logarithmic capacity.

By the Theorem 1 one can find a harmonic function $U: \mathbb{D} \rightarrow \mathbb{R}$ such that

$$
\lim _{w \rightarrow \xi} \frac{\partial U}{\partial \mathcal{N}}(w)=\Phi(\xi)
$$

along any nontangential paths to a.e. point $\xi \in \partial \mathbb{D}$ with respect to logarithmic capacity. Moreover, in the simply connected domain $\mathbb{D}$, one can find a harmonic function $V: \mathbb{D} \rightarrow \mathbb{R}$ such that $g:=U+i \cdot V$ is a single-valued analytic function in $\mathbb{D}$.

Let $F$ be an indefinite integral of the analytic function $g^{\prime} \cdot\left(\omega^{-1}\right)^{\prime}$ in $\mathbb{D}$ and let $f:=F \circ \omega$. Then $F$ and $f$ are also single-valued analytic functions in $\mathbb{D}$ and $D$, correspondingly, and elementary calculations show that

$$
f^{\prime}=F^{\prime} \circ \omega \cdot \omega^{\prime}=F^{\prime} \circ \omega \cdot\left(\omega^{\prime} \circ \omega^{-1}\right) \circ \omega=\left[F^{\prime} /\left(\omega^{-1}\right)^{\prime}\right] \circ \omega=g^{\prime} \circ \omega \text {. }
$$

Thus,

$$
\frac{\partial f}{\partial \nu}=\nu \cdot f^{\prime}=\nu \cdot g^{\prime} \circ \omega=\frac{\partial g}{\partial \mathcal{N}} \circ \omega,
$$

where $\nu=\nu(\zeta), \zeta \in \partial D$, and $\mathcal{N}=\mathcal{N}(\xi), \xi=\omega(\zeta) \in \partial \mathbb{D}$. Hence, for $u:=$ Re $f$, we have the equality

$$
\frac{\partial u}{\partial \nu}=\frac{\partial U}{\partial \mathcal{N}} \circ \omega
$$


and, consequently, $u$ is a desired harmonic function by (8).

By Theorem 3, arguing similarly to Remark 1, we have the following significant result on the Neumann problem.

Theorem 4. Let $D$ be an almost smooth Jordan domain in the complex plane $\mathbb{C}$ and let a function $\varphi: \partial D \rightarrow \mathbb{R}$ be measurable with respect to logarithmic capacity. Then one can find harmonic functions $u: D \rightarrow \mathbb{C}$ such that for a.e. $\zeta \in \partial D$ with respect to logarithmic capacity, there exist:

1) the finite normal limit

$$
u(\zeta):=\lim _{z \rightarrow \zeta} u(z)
$$

2) the normal derivative

$$
\frac{\partial u}{\partial n}(\zeta):=\lim _{t \rightarrow 0} \frac{u(\zeta+t \cdot n)-u(\zeta)}{t}=\varphi(\zeta)
$$

3) the nontangential limit

$$
\lim _{z \rightarrow \zeta} \frac{\partial u}{\partial n}(z)=\frac{\partial u}{\partial n}(\zeta)
$$

\section{On Neumann and Poincare Problems for $A$-harmonic Functions}

Theorem 5. Let $D$ be an almost smooth Jordan domain in $\mathbb{C}, A(z), z \in D$, be a matrix function of the class $\mathcal{B} \cap C^{\alpha}, \alpha \in(0,1), \nu: \partial D \rightarrow \mathbb{C},|\nu(\zeta)| \equiv 1$, be a function of bounded variation and let a function $\varphi: \partial D \rightarrow \mathbb{R}$ be measurable with respect to logarithmic capacity. Then there exist A-harmonic functions $u: D \rightarrow \mathbb{R}$ of the class $C^{1+\alpha}$ such that

$$
\lim _{z \rightarrow \zeta} \frac{\partial u}{\partial \nu}(z)=\varphi(\zeta)
$$

along any nontangential paths for a.e. $\zeta \in \partial D$ with respect to logarithmic capacity.

Proof. By remarks in Section 2, a desired function $u$ is a real part of a solution $f$ of the class $W_{\text {loc }}^{1,1}$ for corresponding Beltrami equation with $\mu \in C^{\alpha}$, see e.g. Theorem 16.1.6 in [1]. By Lemma 1 in [2] $\mu$ is extended to a Hölder continuous function $\mu_{*}: \mathbb{C} \rightarrow \mathbb{C}$ of the class $C^{\alpha}$. Hence also, for every $k_{*} \in(k, 1)$, there is an open neighborhood $U$ of $D_{*}$ such that $|\mu(z)|<k_{*}$. Let $D_{*}$ be a connected component of $U$ containing $\bar{D}$.

Next, there is a quasiconformal mapping $h: D_{*} \rightarrow \mathbb{C}$ a.e. satisfying the Beltrami equation (1) with the complex coefficient $\mu^{*}:=\left.\mu_{*}\right|_{D_{*}}$ in $D_{*}$, see e.g. Theorem V.B.3 in [8]. Note that the mapping $h$ has the Hölder continuous first partial derivatives in $D_{*}$ with the same order of the Hölder continuity as $\mu$, see e.g. [14] and also [15]. Moreover, the mapping $h$ is regular, i.e. its Jacobian

$$
J_{h}(z) \neq 0 \quad \forall z \in D_{*},
$$

see e.g. Theorem V.7.1 in [9]. Thus, the directional derivative

$$
h_{\omega}(z)=\frac{\partial h}{\partial \omega}(z):=\lim _{t \rightarrow 0} \frac{h(z+t \cdot \omega)-h(z)}{t} \neq 0 \quad \forall z \in D_{*} \forall \omega \in \partial \mathbb{D}
$$

and it is continuous by the collection of the variables $\omega \in \partial \mathbb{D}$ and $z \in D_{*}$. Thus, the functions

$$
\nu_{*}(\zeta):=\frac{\left|h_{\nu(\zeta)}(\zeta)\right|}{h_{\nu(\zeta)}(\zeta)} \quad \text { and } \quad \varphi_{*}(\zeta):=\frac{\varphi(\zeta)}{\left|h_{\nu(\zeta)}(\zeta)\right|}
$$

are measurable with respect to logarithmic capacity, see e.g. 17.1 in [16].

The logarithmic capacity of a set coincides with its transfinite diameter, see e.g. [12] and the point 110 in [13]. Moreover, quasiconformal mappings are Hölder continuous on compacta, see e.g. Theorem 
II.4.3 in [9]. Hence the mappings $h$ and $h^{-1}$ transform sets of logarithmic capacity zero on $\partial D$ into sets of logarithmic capacity zero on $\partial D^{*}$, where $D^{*}:=h(D)$, and vice versa.

Further, the functions $\mathcal{N}:=\left.\nu_{*} \circ h^{-1}\right|_{\partial D^{*}}$ and $\Phi:=\left.\left(\varphi_{*} / h_{\nu}\right) \circ h^{-1}\right|_{\partial D^{*}}$ are measurable with respect to logarithmic capacity. Indeed, a measurable set with respect to logarithmic capacity is transformed under the mappings $h$ and $h^{-1}$ into measurable sets with respect to logarithmic capacity because such a set can be represented as the union of a sigma-compactum and a set of logarithmic capacity zero and compacta are transformed under continuous mappings into compacta and compacta are measurable with respect to logarithmic capacity.

Recall that the distortion of angles under quasiconformal mappings $h$ and $h^{-1}$ is bounded, see e.g. $[17,18,19]$. Thus, nontangential paths to $\partial D$ are transformed into nontangential paths to $\partial D^{*}$ for a.e. $\zeta \in \partial D$ with respect to logarithmic capacity and inversly.

By Theorem 3, one can find a harmonic function $U: D^{*} \rightarrow \mathbb{R}$ such that

$$
\lim _{w \rightarrow \xi} \frac{\partial U}{\partial \mathcal{N}}(w)=\Phi(\xi)
$$

along any nontangential paths for a.e. $\xi \in \partial D^{*}$ with respect to logarithmic capacity.

Moreover, one can find a harmonic function $V$ in the simply connected domain $D^{*}$ such that $F=U+i V$ is an analytic function and, thus, $u:=\operatorname{Re} f=U \circ h$, where $f:=F \circ h$, is a desired $A$-harmonic function because $f$ is a regular solution of the corresponding Beltrami equation (1) and also

$$
u_{\nu}=\left\langle\nabla U \circ h, h_{\nu}\right\rangle=\left\langle\nu_{*} \cdot \nabla U \circ h, \nu_{*} \cdot h_{\nu}\right\rangle=\left\langle\frac{\partial U}{\partial \mathcal{N}} \circ h, \nu_{*} \cdot h_{\nu}\right\rangle=\frac{\partial U}{\partial \mathcal{N}} \circ h \cdot \operatorname{Re}\left(\nu_{*} h_{\nu}\right),
$$

i.e. condition (9) holds along any nontangential paths for a.e. $\zeta \in \partial D$ with respect to logarithmic capacity.

The following statement concerning the Neumann problem for $A$-harmonic functions is a special significant case of Theorem 5 .

Theorem 6. Let $D$ be an almost smooth Jordan domain in $\mathbb{C}$, the interior unit normal $n=n(\zeta)$ to $\partial D$ have bounded variation, $A(z), z \in D$, be a matrix function of class $\mathcal{B} \cap C^{\alpha}, \alpha \in(0,1)$ and let a function $\varphi: \partial D \rightarrow \mathbb{R}$ be measurable with respect to logarithmic capacity. Then there exists an A-harmonic function $u: D \rightarrow \mathbb{R}$ of class $C^{1+\alpha}$ such that for a.e. $\zeta \in \partial D$ with respect to logarithmic capacity there exist:

1) the finite normal limit

$$
u(\zeta):=\lim _{z \rightarrow \zeta} u(z)
$$

2) the normal derivative

$$
\frac{\partial u}{\partial n}(\zeta):=\lim _{t \rightarrow 0} \frac{u(\zeta+t \cdot n)-u(\zeta)}{t}=\varphi(\zeta)
$$

3) the nontangential limit

$$
\lim _{z \rightarrow \zeta} \frac{\partial u}{\partial n}(z)=\frac{\partial u}{\partial n}(\zeta)
$$

In particular, in the unit disk $\mathbb{D}$, the unit interior normal $n=n(\zeta)$ to $\partial \mathbb{D}$ has bounded variation and, thus, the conclusions 1-3 of the latter theorem hold.

\section{On the Dimension of Spaces of Solutions}

Theorem 7. The spaces of solutions in Theorems 1-6 have the infinite dimension.

Proof. In view of the equivalence of the problem on the directional derivatives to the corresponding Riemann-Hilbert boundary value problem established under the proof of Theorem 1, the conclusion of Theorem 7 follows from Theorem 8.2 in [6] because Theorems 2-6 are successively reduced to Theorem 1.

Acknowledgement. I would like to thank Professor Vladimir Ryazanov for his scientific supervision. 


\section{References}

1. K. Astala, T. Iwaniec, and G. Martin, Elliptic partial differential equations and quasiconformal mappings in the plane. Princeton University Press, 2009, vol. 48.

2. V. Gutlyanskii, V. Ryazanov, and A. Yefimushkin, "On the boundary-value problems for quasiconformal functions in the plane," Journal of Mathematical Sciences, vol. 214, no. 2, pp. 200-219, 2016.

3. V. Ryazanov, "On the Riemann-Hilbert problem without Index," Ann. Univ. Bucharest, Ser. Math, vol. 5, pp. 169-178, 2014.

4. - "Infinite dimension of solutions of the Dirichlet problem," Open Math.(the former Central European J. Math.), vol. 13, pp. 348-350, 2015.

5. —_ "On Neumann and Poincare problems for Laplace equation," Analysis and Mathematical Physics, pp. 1-5, 2016. [Online]. Available: http://dx.doi.org/10.1007/s13324-016-0142-8

6. A. Yefimushkin and V. Ryazanov, "On the Riemann-Hilbert problem for the Beltrami Equations," Contemporary Mathematics, vol. 667, pp. 299-316, 2016.

7. S. G. Mikhlin, Partielle Differentialgleichungen in der mathematischen Physik. Akademie-Verlag, Berlin, 1978.

8. L. V. Ahlfors, Lectures on quasiconformal mappings. Van Nostrand, 1966.

9. O. Lehto and K. Virtanen, Quasiconformal mappings in the plane. Springer-Verlag, 1973, vol. 126.

10. G. M. Goluzin, Geometric theory of functions of a complex variable. Amer. Math. Soc., Providence, 1969.

11. P. Koosis, Introduction to Hp spaces. Cambridge University Press, 1998, vol. 115.

12. M. Fekete, "Über die Verteilung der Wurzeln bei gewissen algebraischen Gleichungen mit ganzzahligen Koeffizienten," Mathematische Zeitschrift, vol. 17, no. 1, pp. 228-249, 1923.

13. R. Nevanlinna, Eindeutige analytische Funktionen. Ann Arbor, Michigan, 1944.

14. T. Iwaniec, "Regularity of solutions of certain degenerate elliptic systems of equations that realize quasiconformal mappings in n-dimensional space," Differential and Integral Equations. Boundary-Value Problems, pp. 97-111, 1979.

15. — , "Regularity theorems for solutions of partial differential equations for quasiconformal mappings in several dimensions," Dissertationes Math. (Rozprawy Mat.), 1982.

16. M. Krasnoselskii, P. Zabreiko, E. Pustylii, and P. Sobolevskii, Integral operators in spaces of summable functions. Noordhoff International Publishing, Leiden, 1976.

17. S. Agard, "Angles and quasiconformal mappings in space," Journal d'Analyse Mathématique, vol. 22, no. 1, pp. 177-200, 1969.

18. S. Agard and F. Gehring, "Angles and quasiconformal mappings," Proceedings of the London Mathematical Society, vol. 3, no. 1, pp. 1-21, 1965.

19. O. Taari, "Charakterisierung der Quasikonformitat mit Hilfe der Winkelverzerrung," Annales Academiae Scientiarum Fennicae, Ser. A, vol. 390, pp. 1-43, 1966. 